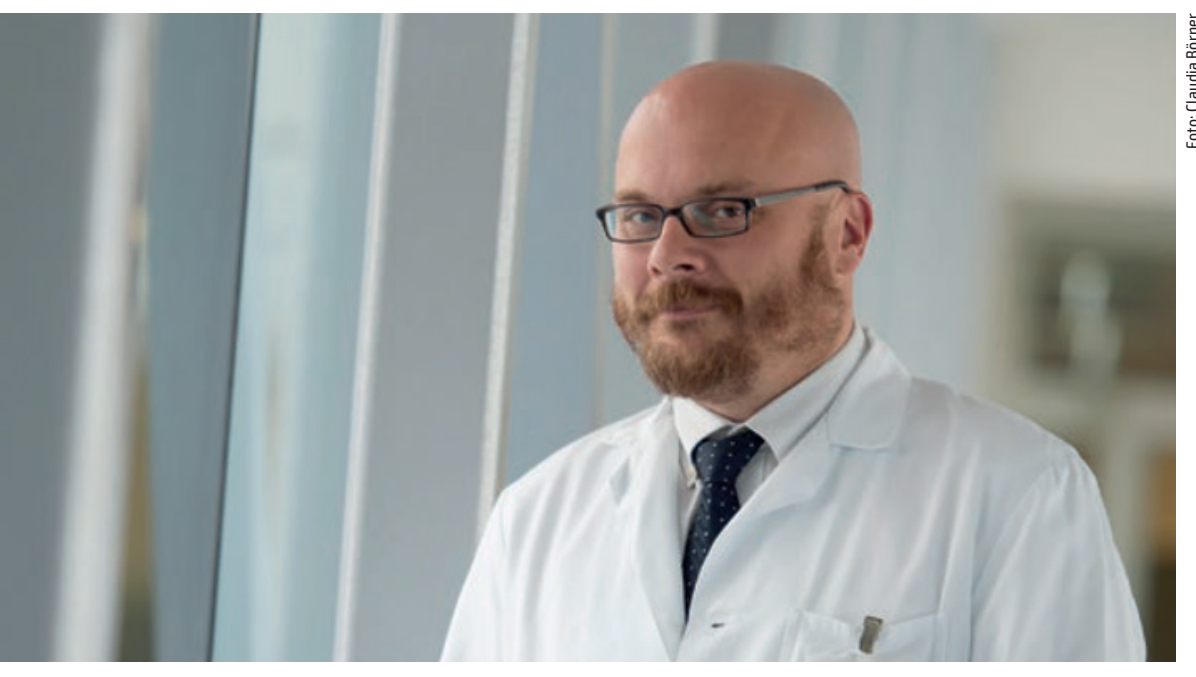

\section{Zur Person.}

Univ.-Prof. Dr. Jens Meier ist seit

Dezember 2019 Vorstand der

Universitätsklinik für Anästhesiologie und Operative Intensivmedizin am Kepler Universitätsklinikum. Im März 2020 wurde der gebürtige Bayer als Koordinator aller intensivmedizinischen Kapazitäten in Oberösterreich eingesetzt.

\title{
„Wann können wir noch von Intensivmedizin reden?"
}

\begin{abstract}
Herr Prof. Meier, Sie wurden von der Landesregierung zum Koordinator aller Kapazitäten bei oberösterreichischen Intensivbetten berufen. Wird Covid im Herbst wieder die oberösterreichischen Spitäler fluten?
\end{abstract}

Jens Meier: Aktuell (Stand Mitte September, Red) sind die Infektionszahlen wieder leicht im Sinken. Allerdings kann es mit schlechtem Wetter und vermehrter Indoor-Aktivität wieder zu einem stärkeren Anstieg kommen. Die Situation ist heute aber anders als vor einem Jahr, als wir im Frühjahr wirklich unter Druck gekommen sind.

\section{Was ist heute so unterschiedlich?}

Die Impfungen verändern die Situation zum Positiven. Vor einem Jahr mussten wir mit einer deutlich höheren Anzahl an hospitalisierten und intensivmedizinisch zu betreuenden Patienten rechnen. Zwar nimmt die relative Zahl an intensivpflichtigen COVID-PatientInnen zu, die Gesamtzahl ist aber durch die Impfung und den damit einhergehenden Rückgang der Erkrankungszahlen geringer. Aktuell sind wir zuversichtlich, nicht wieder bis an die Grenzen gefordert zu werden. Vergangenen Jänner und Februar standen wir kurz vor der Notwendigkeit zu triagieren.
Eine Systemfrage: Warum wurden die inten-
sivmedizinischen Kapazitäten in den vergan-
genen 18 Monaten nicht stärker ausgebaut?

Das ist kurzfristig betrachtet keine Frage des Wollens und auch nicht des Geldes. Intensivmedizin verlangt nach hochkomplexer medizinischer und pflegerischer Kompetenz. Man kann es auch so formulieren: Es gibt das Personal nicht, das wir für einen deutlichen Ausbau der Kapazitäten bräuchten. Intensivmedizin ist stets in Verbindung mit allen anderen Aufgaben eines Krankenhauses zu sehen. Wir müssen die Frage beantworten, wie viele Intensivbetten ungenutzt für den worst case vorgehalten werden. Dafür muss an anderer Stelle reduziert werden. Wo soll dies sein?

In einer Presseaussendung der zuständigen oberösterreichischen Landesrätin Haberlander heißt es anlässlich Ihrer Berufung als Koordinator, das Bundesland könne je nach COVID-Bedrohungslage „in mehreren Schritten auf 270, 358 bzw. sogar 420 Intensivbetten erweitern". Man kann also doch ausbauen?

Zugegebenermaßen ist der Stufenplan auf den ersten Blick verwirrend. Das Problem liegt in der Zählweise: Es gilt zu definieren, was als Intensivbett zu zählen ist. Wir haben in Oberösterreich eine nach dem ÖSG vorgegebene Summe an Intensivbetten, bei denen eine 100-prozentige intensivmedizinische Betreuung nach allen Standards möglich ist. Jetzt haben wir in der speziellen Situation wie der Pandemie aber die Notwendigkeit, bei verschobener oder verringerter Betreuungsleistung mehr Betten zu mobilisieren.

\section{Wie soll dies gehen?}

Es ist eine Frage der Verteilung: Eine Möglichkeit ist es, OP-Säle zu schließen und die Anästhesiepflegekräfte im Intensivbereich einzusetzen. Wir reduzieren also die normale Versorgung. Eine zweite Möglichkeit ist, dass eine Pflegekraft nicht mehr für ein oder zwei Betten verantwortlich ist, sondern für vier, weil in anderen Bereichen, z.B. dem Aufwachraum, zusätzliche Kapazitäten geschaffen werden. So ergeben sich nach unten abgestufte Versorgungsszenarien, mit denen mehr Patienten mit denselben Personalkapazitäten betreut werden. Und dann sind wir bei der Kernfrage, die ich auch in meinem Vortrag am Österreichischen Gesundheitswirtschaftskongress stellen werde: Wie weit können wir unsere Intensivkapazitäten erweitern, ohne den Anspruch an Intensivmedizin aufzugeben? Wann können wir überhaupt noch von Intensivmedizin reden? Da gibt es unterschiedliche Antworten. // 